\title{
Evaluating the health benefits of fruits for physical fitness: A research platform
}

\author{
Birgit Schrage $^{\mathrm{a}}$, David Stevenson ${ }^{\mathrm{a}}$, Robyn W. Wells ${ }^{\mathrm{a}}$, Kirsty Lyall ${ }^{\mathrm{a}}$, Selena Holmes ${ }^{\mathrm{a}}$, \\ Dawei Deng ${ }^{\mathrm{a}}$ and Roger D. Hurst ${ }^{\mathrm{b}, *}$ \\ ${ }^{a}$ Food \& Wellness Group, The New Zealand Institute for Plant \& Food Research Ltd, Hamilton, New Zealand \\ ${ }^{\mathrm{b}}$ Food \& Wellness Group, The New Zealand Institute for Plant \& Food Research Ltd, Palmerston North, New Zealand
}

\begin{abstract}
To evaluate the health promoting attributes of fruits and their compounds the New Zealand Institute for Plant \& Food Research Ltd (PFR) is using exercise as a model for oxidative stress and immune depression. Regular exercise has health benefits believed to be derived from adaptive responses to moderate oxidative stress. However, following exhaustive or unaccustomed exercise, excessive and prolonged oxidative stress and inflammation can be detrimental and the right balance of modulation from nutritional support via fruit phytochemicals (and vitamins) may prevent damage, aid recovery, and/or enhance muscular and immune function. We have developed a research platform to evaluate physical health, performance and recovery to position new fruit varieties in this area. Utilising compositional analysis of fruit extracts, in vitro screening of muscle cells, electrically stimulated muscle ex vivo, and animal and human intervention and exercise trials, we are evaluating the physical health-promoting effects of polyphenolic phytochemicals derived from fruit, particularly berry fruits. Our research demonstrates that certain fruits may complement the benefits of regular exercise through appropriate modulation of excessive oxidative stress and inflammation. Keywords: Berry fruit, kiwifruit, fruit, polyphenolics, phytochemicals, anthocyanins, oxidative stress, inflammation, exercise, skeletal muscle
\end{abstract}

\section{Introduction}

The New Zealand Institute for Plant \& Food Research Ltd (PFR) provides research and innovation for New Zealand's horticultural sector. PFR has developed a research platform to evaluate physical performance and recovery. Regular exercise has health benefits derived from moderate oxidative stress. However, depending on the mode, intensity and duration, exercise can inflict high levels of mechanical and metabolic stress on muscle [28]. Dietary supplementation is an attractive choice for health-conscious individuals. However, the right balance of modulation from nutritional support needs to be established to prevent damage/injury, maximise recovery for enhanced muscle function, but also to optimise immune function.

Utilising fruit varieties from breeding programmes at PFR with well-defined compositional data combined with a range of cell-based screening, ex vivo muscle experiments, and animal/human trials, we are evaluating the physical health-promoting effects of phytochemicals derived from some new fruit varieties, including berry fruits. The determination of biochemical markers of oxidative stress, inflammation and immune function in cell and tissue assays, and animal and human trials enables us to evaluate the effects of fruit consumption and to ultimately guide the breeding of health promoting fruit.

*Corresponding author: Roger D. Hurst, Food \& Wellness Group, The New Zealand Institute for Plant \& Food Research Ltd, Private Bag 11600, Palmerston North 4442, New Zealand. Tel.: +64 6355 6203; Fax: +64 6351 7050; E-mail: Roger.Hurst@plantandfood.co.nz. 


\section{Materials and methods}

\subsection{Fruit extract preparation and composition}

The ZESPRI ${ }^{\circledR}$ GOLD kiwifruit (Actinidia chinensis 'Hort16A') extract was prepared by homogenising freeze-dried fruit $(20 \mathrm{~g})$ with methanol $(50 \mathrm{~mL})$, followed by $30 \mathrm{~min}$ in an ultrasonic bath. Solvent was recovered by filtration and the residue extracted twice more in methanol. The extract was rotary evaporated, dissolved in the minimum volume of water and applied to a $10 \mathrm{~g} \mathrm{C}-18$ solid-phase extraction cartridge (Strata, Phenomenex, Torrance, CA). The cartridge was flushed with deionised water $(30 \mathrm{~mL})$ and eluted with methanol $(40 \mathrm{~mL})$. Evaporation of the methanol eluate yielded $0.24 \mathrm{~g}$ of extract which was prepared in buffer for the analysis of ex vivo muscle performance.

The polyphenolic extract of blueberry fruit (Vaccinium corymbosum, 'Reka') was prepared from approximately $800 \mathrm{~g}$ of frozen fruit homogenised with acetone and the residue recovered by filtration. Following a further homogenisation and filtration with acetone/water (70:30), acetone extracts were combined and concentrated by rotary evaporation. Lipids were removed by partitioning the aqueous extract with heptane and the residual heptane in the aqueous layer removed by rotary evaporation. The polyphenolics were isolated from this extract by absorption, recovery, and elution (methanol) of XAD-7 (Sigma, Sydney, Australia) and dried by rotary evaporation to yield a friable powder. In all experiments, the blueberry fruit extracts were prepared from a stock in DMSO. DMSO concentrations in the final experimental conditions were below $1 \%$ and had no observable effect.

Juices of various cultivars of blackcurrant (Ribes nigrum) were prepared by blending fruit ( $180 \mathrm{~g}$ ) with a domestic hand blender, then treating with Pectinex $(0.18 \mathrm{~mL}$; Novozymes, Bagsvaerd, Denmark $)$ at $48^{\circ} \mathrm{C}$ for $4 \mathrm{~h}$. After centrifugation (4000 rpm, $10 \mathrm{~min}$ ) the yield of juice was typically $\sim 50 \%$. Blackcurrant juice samples were analysed by liquid chromatography-mass spectrometry (LC-MS) using a Shimadzu (Auckland, New Zealand) 20-Series UFLC system including an autosampler, column oven and photodiode-array detector, linked to an LCMS 2020 single-quadrupole mass spectrometer. The LC system was fitted with a $150 \times 2 \mathrm{~mm}, 4 \mu$ Synergi Fusion RP column (Phenomenex). For analysis, blackcurrant samples were diluted $\times 10$ into $1 \%$ aqueous formic acid and kiwifruit extracts dissolved in water at $\sim 2 \mathrm{mg} / \mathrm{mL}$. Samples of $10 \mu \mathrm{L}$ were injected and eluted by the following solvent programme. Flow rate $0.6 \mathrm{~mL} / \mathrm{min}$, Solvent A: $2 \%$ formic acid, solvent B: methanol. The initial conditions were $8 \%$ B and ramped linearly to $10 \%$ at $2.5 \mathrm{~min}, 18 \%$ at $5 \mathrm{~min}, 38 \%$ at $8.5 \mathrm{~min}, 63 \%$ at $10.5 \mathrm{~min}$, held at $70 \%$ between 11.5 and $12.5 \mathrm{~min}$, then back to the starting conditions between 13 and $15 \mathrm{~min}$. Column oven temperature was $50^{\circ} \mathrm{C}$. The mass spectrometer was operated under standard tuning conditions, as specified by the manufacturer and half the LC flow was diverted to waste through a split valve. Identification for compounds of interest was achieved by comparison of a combination of UV-visible and mass spectra with standard compounds.

The commercially available juices GHO "Natural Quenchers" (Good Health Organisation, Nelson, New Zealand) contained $65 \%$ of either ZESPRI ${ }^{\circledR}$ GOLD kiwifruit or blackcurrant juice.

\subsection{Determination of muscle cell intracellular free radical levels}

The rat skeletal muscle cell line L6 (American Type Culture Collection, Manassas, USA) was cultured and differentiated as described elsewhere [13]. Muscle cells were plated at 5000 cells/well in 96 well plates and incubated with $2^{\prime}, 7^{\prime}$-dichlorohydrofluorescein diacetate (DCFDA) $(10 \mu \mathrm{M})$ for $60 \mathrm{~min}$ at room temperature. DCFDA is cleaved intracellularly into DCF, which produces an increase in fluorescence in the presence of reactive free radicals. Following a wash in D-PBS $(2 \times)$, cells were equilibrated at room temperature for $10 \mathrm{~min}$ prior to incubation (simultaneously [time zero] and at the times indicated) with the blueberry fruit extract $(50 \mu \mathrm{g} / \mathrm{mL})$, followed by exposure to $\mathrm{H}_{2} \mathrm{O}_{2}$ $(0.5 \mathrm{mM})$. Fluorescence intensity was determined over time using a fluorescence platereader (BMG FluorStar Optima, Alphatech Systems Ltd) with excitation and emission wavelengths of 485 and $520 \mathrm{~nm}$ respectively.

\subsection{Evaluation of muscle cell total glutathione (GSH) levels and glutathione peroxidase (GPx) activity}

The total amount of muscle cell GSH and GPx was determined colourimetrically using Bioxytech ${ }^{\circledR}$ assay kits (GSH-420 ${ }^{\mathrm{TM}}$ and GPx-340 ${ }^{\mathrm{TM}}$, Sapphire Bioscience Pty. Ltd, Auckland). Differentiated L6 myotubes were incubated with the blueberry fruit extract both simultaneously and for $24 \mathrm{~h}$ before GSH and GPx determination. For GSH 
analysis, cells were plated at a density of $1 \times 10^{6}$ cells/well in 6 well plates and grown until confluent. Following extract exposure, cells were harvested using Tryple ${ }^{\mathrm{TM}}$, washed in D-PBS, and permeabilised by sonication in the precipitation reagent provided by the kit. All other analysis steps were then as recommended by the supplier. To determine muscle cell GPx levels, cells were grown in T75 flasks until confluent. Following extract exposure, cells were harvested using a cell scraper and prepared into $3 \times 10^{6}$ cell aliquots before sonication in D-PBS containing $1 \mathrm{mM}$ mercaptoethanol. All other analysis steps were as recommended by the supplier.

\subsection{Animals}

Male Swiss mice, 8-12 weeks old, were used for the muscle bath and animal exercise experiments. Animals were cared for in accordance with the Code of Ethical Conduct, Regulations Act, 1987 (New Zealand) and all procedures were approved by our local Animal Ethics Committee.

\subsection{Ex vivo model of muscle performance}

To investigate the effect of fruit extracts on muscle tissue, a model bath system was established in which isolated fresh muscle (Soleus) was exposed to extracts and subsequently electrically stimulated to examine the effects on contractile activity (both maximum force produced and fatigue profiles).

Animals were killed by $\mathrm{CO}_{2}$ asphyxiation. Soleus muscles were isolated from both hindlimbs, leaving tendons attached. Muscles were placed in an organ bath in Krebs-Ringer solution (in mM: $118 \mathrm{NaCl}, 4.75 \mathrm{KCl}, 1.18 \mathrm{MgSO}_{4}$, $2.54 \mathrm{CaCl}_{2}, 24.8 \mathrm{NaHCO}_{3}, 1.18 \mathrm{KH}_{2} \mathrm{PO}_{4}, 10$ glucose), bubbled continuously with $95 \% \mathrm{O}_{2}-5 \% \mathrm{CO}_{2}$ to maintain a pH of 7.5 at $25^{\circ} \mathrm{C}$. Muscles were attached to a force transducer (World Precision Instruments, Sarasota, USA) and positioned between electrodes. Using a micrometer, muscles were adjusted to their optimum length to yield maximal force at $120 \mathrm{~Hz}$ stimulation. The output of the force transducer was monitored using Data-Trax software (World Precision Instruments, Sarasota, USA).

Baseline tetanic force production (3 millisecond pulses at $20 \mathrm{~Hz}$ over $14 \mathrm{~s}$ ) in each individual muscle was measured in the absence of fruit extract exposure. After a recovery period of $10 \mathrm{~min}$, the buffer was replaced with fresh KrebsRinger solution for control muscles, or Krebs-Ringer solution containing $5 \mu \mathrm{g} / \mathrm{mL}$ ZESPRI ${ }^{\circledR}$ GOLD kiwifruit extract or $2 \%$ GHO "Natural Quenchers" containing 65\% of either ZESPRI ${ }^{\circledR}$ GOLD kiwifruit or blackcurrant juice. Muscles were incubated for $15 \mathrm{~min}$ before stimulating another tetanus response. Muscles were then removed and length and weight measured; the cross sectional area was estimated according to Mendez and Keys [19] and force expressed as $\mathrm{mN} / \mathrm{mm}^{2}$. Force measured during the fatigue protocols was assessed in relation to the peak force generated in each individual muscle's baseline stimulation.

\subsection{Animal exercise}

Mice were randomly assigned to the following five groups: no-exercise controls, exercising mice sacrificed at $0,1,3$, or $24 \mathrm{~h}$ after fatiguing uphill running. All mice were acclimated to exercise conditions on the motorized treadmill (Muromachi Kikai, Tokyo, Japan) for five days, during which they walked/ran for $15 \mathrm{~min}$ at a speed of $0-15$ metres/min and a maximum incline of $+2^{\circ}$. The running adaptation was followed by at least five days of rest. During the fatiguing exercise, mice ran on the treadmill with a maximum speed of $36 \mathrm{metres} / \mathrm{min}$ and a maximum incline of $+8^{\circ}$ until the point of fatigue, at which they were no longer able to maintain pace despite gentle prodding and discharges of compressed air. Control mice were kept in the room with the treadmill to expose them to the same noise and handling as the exercising mice. After completion of the exercise, mice in the control and 0 hour group were sacrificed immediately, all other mice were sacrificed after 1, 3, or $24 \mathrm{~h}$ of recovery. Animals were killed by $\mathrm{CO}_{2}$ asphyxiation and blood was collected by heart puncture. Blood was centrifuged $(9000 \mathrm{~g}, 10 \mathrm{~min})$ and plasma was stored at $-80^{\circ} \mathrm{C}$ until further analysis. Plasma creatine kinase (CK) activity was analysed by Gribbles Veterinary Laboratory (Hamilton, New Zealand). Results are expressed as international units per litre of plasma (IU/L). Plasma with a haemolysis index greater than 60 was excluded from the analysis. Interleukin-6 (IL-6) concentration in plasma was analysed using the Quantikine Mouse IL-6 ELISA from R\&D Systems (Minneapolis, 
USA) and was performed according to the manufacturer's instructions. Results are expressed as pg IL-6 per mL plasma $(\mathrm{pg} / \mathrm{mL})$.

\subsection{Statistical analysis}

Results are expressed as means \pm standard errors of the mean (SEM) for at least four observations in each case. Statistical significance for the comparison of two groups was assessed by the Student's $t$-test. A probability value $(P)$ of less than 0.05 was considered significant.

\section{Results and discussion}

\subsection{Anthocyanin content of fruit juices and effects of fruit on muscle cell oxidative stress}

Many fruits, and especially berries, contain bioactive compounds whose health-promoting properties are widely recognised [1, 27]. Utilising fruit varieties from breeding programmes at PFR with compositional analysis we are evaluating the physical health-promoting effects of phytochemicals derived from some new fruit varieties, including berry fruits. Breeding programmes at PFR have targeted high anthocyanin content as one of the possible desirable characteristics of new fruit cultivars for health benefits. A compositional survey of juices prepared from commercially grown non-New Zealand as well as New Zealand blackcurrant cultivars and new selections from the PFR breeding programmes demonstrates that the anthocyanin content of the New Zealand fruit juice is approximately 1.5 times that of the non-New Zealand and one new cultivar is particularly high in anthocyanins (Table 1). The fruits for the juices were grown under New Zealand conditions and the data suggest that New Zealand cultivars perform well when compared with those grown in North America [20]. The exceptionally high anthocyanin content makes some New Zealand blackcurrants cultivars unique and might provide potential strategies to counter/modulate the stress and damage associated with over-exercise, as well as to improve overall body wellness.

Cellular studies can be used to determine the underlying mechanisms of action of active fruit extracts/compounds. We report here insights into the protective mechanism of action of a blueberry fruit extract. We analysed the antioxidant ability of skeletal muscle myotubes (differentiated from muscle myoblast cell lines) following exposure to the extract. Using DCFDA, which accumulates intracellularly and is cleaved to produce the radical sensitive product DCF, enabled the determination of intracellular free radical levels by monitoring of relative fluorescence intensities after exposure to $\mathrm{H}_{2} \mathrm{O}_{2}$. Figure 1 displays the antioxidant ability of muscle cells following different lengths of incubation exposure

Table 1

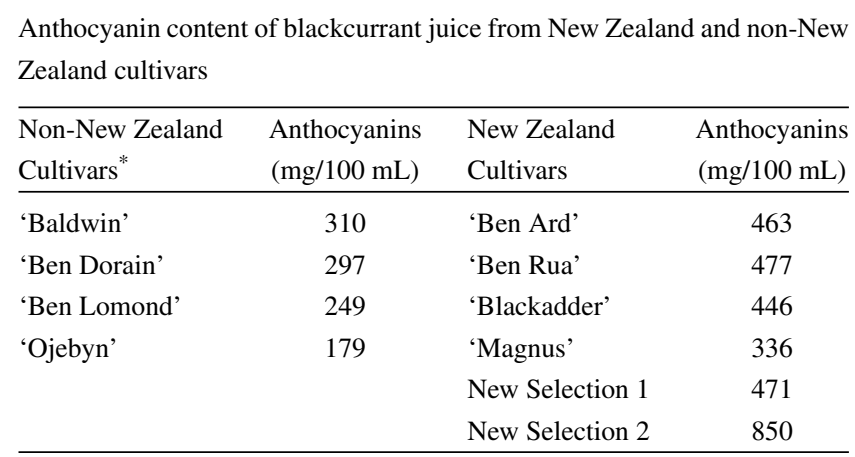

Juices were made from fruit harvested in the 2009 season from bushes on research plots in Canterbury, New Zealand.

*A comparable range of anthocyanin content was obtained from the large study carried out on 32, mostly European, blackcurrant fruit cultivars grown in North America [20]. 

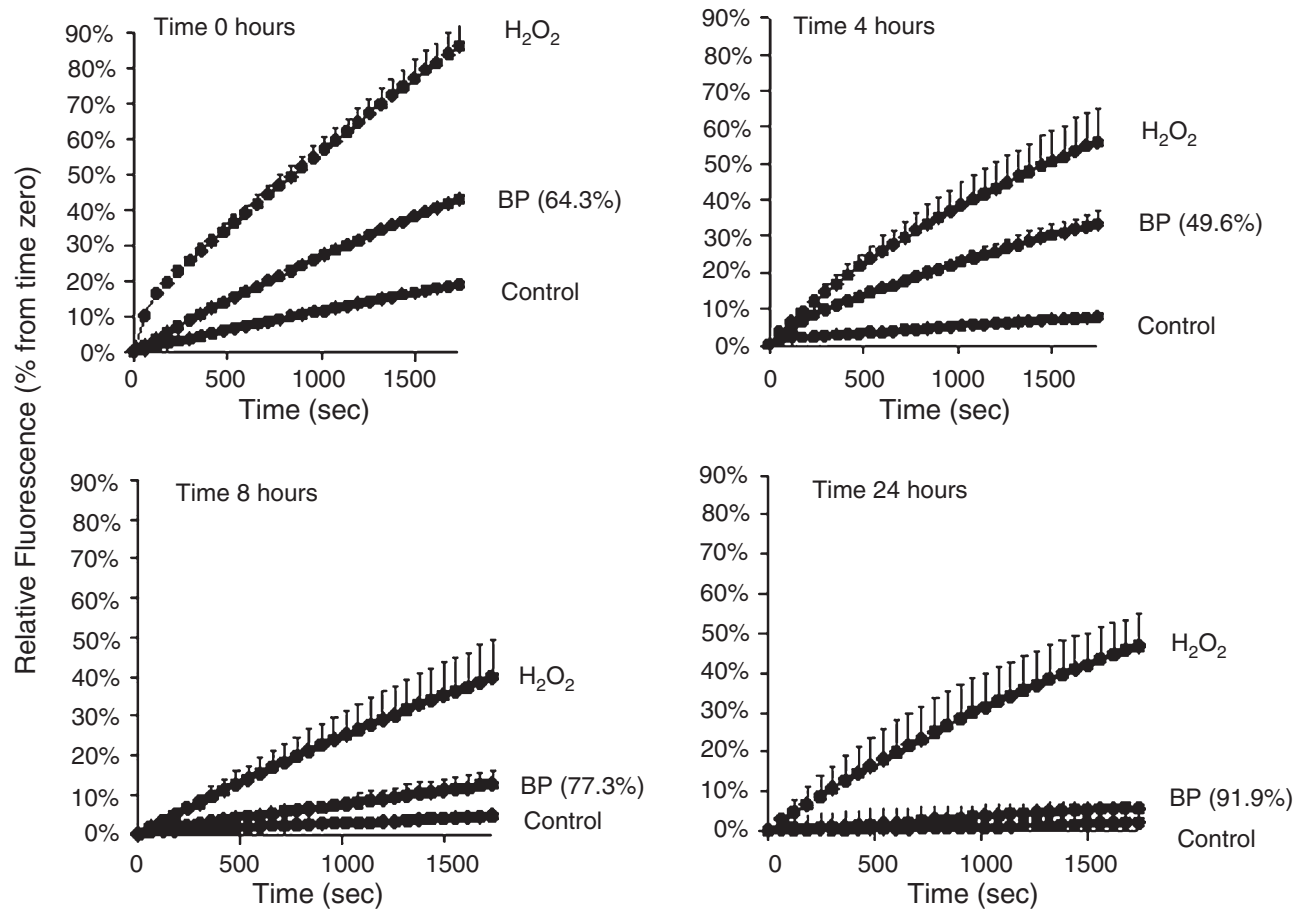

Fig. 1. Effect of incubation time on the intracellular free radical scavenging ability of muscle cells following exposure to a blueberry polyphenolic extract. Muscle cells were loaded with the reactive oxygen species indicator DCF as described in Materials and Methods and incubated (simultaneously [time zero] and at the times indicated on the figures) with a blueberry fruit (Vaccinium corymbosum 'Reka') polyphenolics extract (BP, $50 \mu \mathrm{g} / \mathrm{mL}$ ) prior to challenge with $\mathrm{H}_{2} \mathrm{O}_{2}$ and fluorescence intensity determination. Values are means $\pm \mathrm{SEM}$ from at least four experiments and are expressed as relative fluorescence intensity (\% from time zero). The percentage inhibition of $\mathrm{H}_{2} \mathrm{O}_{2}$-induced fluorescence by the blueberry extract is shown in parentheses.

(simultaneously [time zero] to $24 \mathrm{~h}$ ) with the blueberry extract $\left(50 \mu \mathrm{g} / \mathrm{ml}\right.$ ) before oxidative challenge. $\mathrm{H}_{2} \mathrm{O}_{2}$ elicited a sustained, progressive, and large increase in muscle intracellular free radical levels. The magnitude of this response was reduced from $4 \mathrm{~h}$ onwards and was probably due to the leakage of the DCF from the cells over time, but this did not affect the determination of the intracellular antioxidant ability. With the simultaneous incubation of the muscle cells with the blueberry fruit extract, a marked increase in cellular antioxidant ability was observed, which was reflected by a 64.3\% inhibition of fluorescence signal elicited by $\mathrm{H}_{2} \mathrm{O}_{2}$. This immediate antioxidant ability was further enhanced by longer extract pre-incubations (Fig. 1). After a 24-h extract pre-incubation, the antioxidant scavenging ability of the muscle cells had increased and was reflected by a $91.9 \%$ inhibition of the fluorescence signal elicited by $\mathrm{H}_{2} \mathrm{O}_{2}$. We further evaluated whether washing the blueberry extract from the cells prior to challenge with $\mathrm{H}_{2} \mathrm{O}_{2}$ prevented the immediate and longer-term antioxidant effects. There was no significant difference between washed and non-washed cells (data not shown).

In vitro models of oxidative stress using skeletal muscle cells have been used successfully in the past to monitor, under controlled conditions, the responses of muscle to oxidative stress that cannot be easily evaluated in human trials, and to provide a cost-effective method for the screening of beneficial substances. A useful and simple system is the use of skeletal muscle myotubes (differentiated from muscle myoblast cell lines) exposed to calcium ionophores [17].

We have previously demonstrated that the blueberry fruit (Vaccinium corymbosum 'Reka') polyphenolic extract mediated protection against muscle cell oxidative stress and damage [13]. The data presented here give insights into the potential process of action. We compared the functional activity of anthocyanin rich sub-extracts derived from the total extract with individual anthocyanin components and demonstrated that the most likely actives responsible 
for the protection against the oxidative stress were malvidin glycosides, particularly malvidin galactoside, and/or malvidin glucoside [13].

\subsection{Effects on muscle cell GSH and GPx activity}

The data in Fig. 1 suggest that while there is an immediate antioxidant ability of the fruit extract, the increase in antioxidant ability over time is not associated with the extract itself. Tissues and cells are equipped with efficient enzymatic and non-enzymatic antioxidant defence systems, which serve to counter the damaging action of oxidative stress [10]. Figure 2 shows the determination of muscle cell GSH and GPx activity following blueberry fruit extract exposure. While there were no changes in GPx activity, GSH levels after a 24-h exposure were significantly enhanced and suggest the induction of endogenous antioxidant pathways. These data support other reports that highlight that polyphenolics can modulate the levels of antioxidant defence enzymes such as superoxide dismutase, catalase, GSH and GPx [29]. Evidence suggests that these adaptive actions to ensure cell and tissue survival following oxidative stress may be mediated by the induction of Nuclear factor (erythroid-derived 2)-like 2 (Nrf2). Nrf2 is a transcription factor that binds to the antioxidant response element (ARE) in the upstream promoter region of many antioxidative genes, where it initiates the transcription of many cytoprotective proteins including endogenous antioxidant enzymes [15, 22]. More research is warranted to elucidate the significance of fruit polyphenolic-mediated induction of endogenous antioxidant pathways for modulation of unregulated oxidative stress and the potential long-term effects for muscle performance.

\subsection{Effect of fruit on muscle function}

Exposure of isolated muscle tissue to some fruit extracts also modulates muscle tissue contractile activity and performance. Assessing force production and fatigue profile in electrically stimulated murine soleus muscles, we have previously reported [26] that a ZESPRI ${ }^{\circledR}$ GOLD kiwifruit extract mediated an increased maximum tetanic force in a similar manner to the antioxidant superoxide dismutase $(1000 \mathrm{IU} / \mathrm{mL})$.

Here we report (Fig. 3) the contractile performance of soleus muscle following exposure to a ZESPRI ${ }^{\circledR}$ GOLD Kiwifruit extract and two commercially prepared ZESPRI ${ }^{\circledR}$ GOLD kiwifruit and blackcurrant juices (2\% GHO
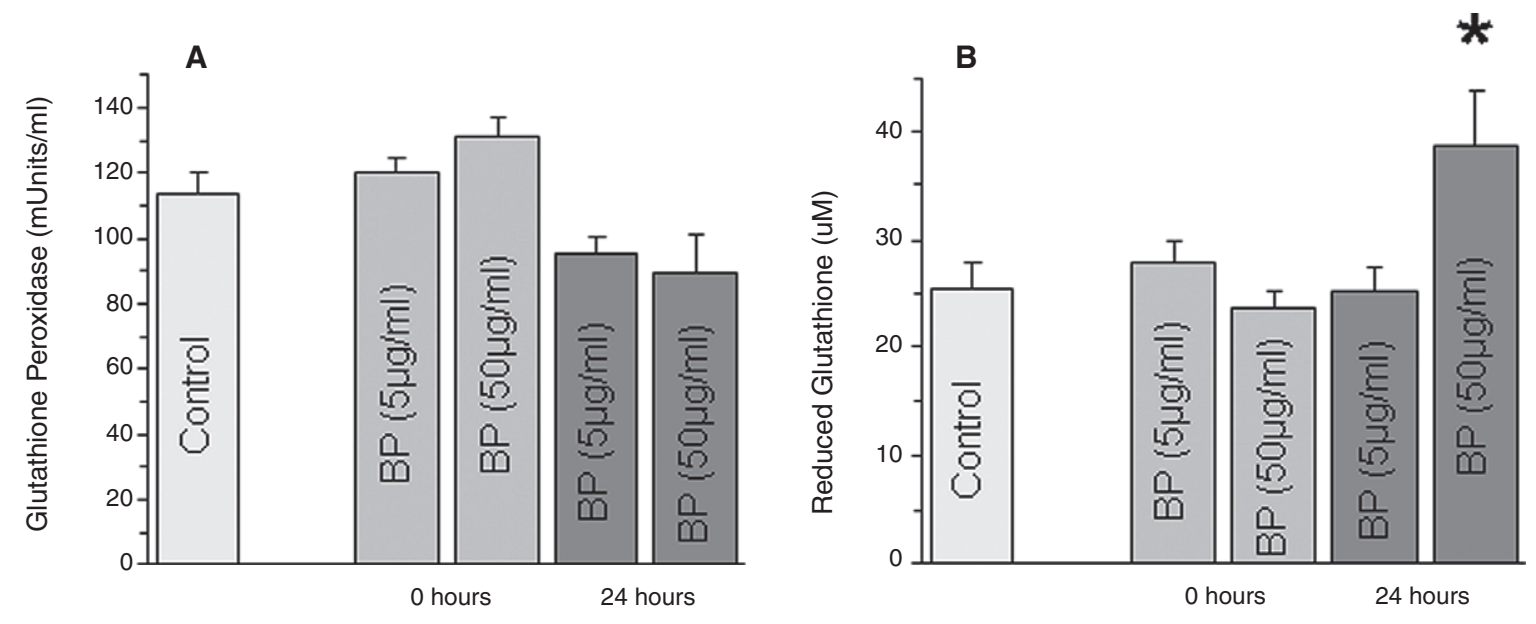

Fig. 2. Muscle cell reduced glutathione levels and glutathione peroxidase activity following incubation with a blueberry fruit polyphenolic extract. The effect of a blueberry (Vaccinium corymbosum 'Reka') polyphenolics extract (BP, 5 and $50 \mu \mathrm{g} / \mathrm{mL}$ ) on muscle cell glutathione peroxidase (GPx) activity and reduced glutathione (GSH) levels was evaluated. Muscle cell GPx (A) and GSH levels (B) were determined $\square$ immediately and $24 \mathrm{~h}$ after incubation with the blueberry extract (BP) as described in Materials and Methods. Values are means \pm SEM from at least four experiments and are expressed as $\mathrm{mU} / \mathrm{mL}$ and $\mu \mathrm{M}$ (GPx and GSH respectively). *Represents $p<0.05$ statistical significance (paired Student $t$-test) compared with the control. 

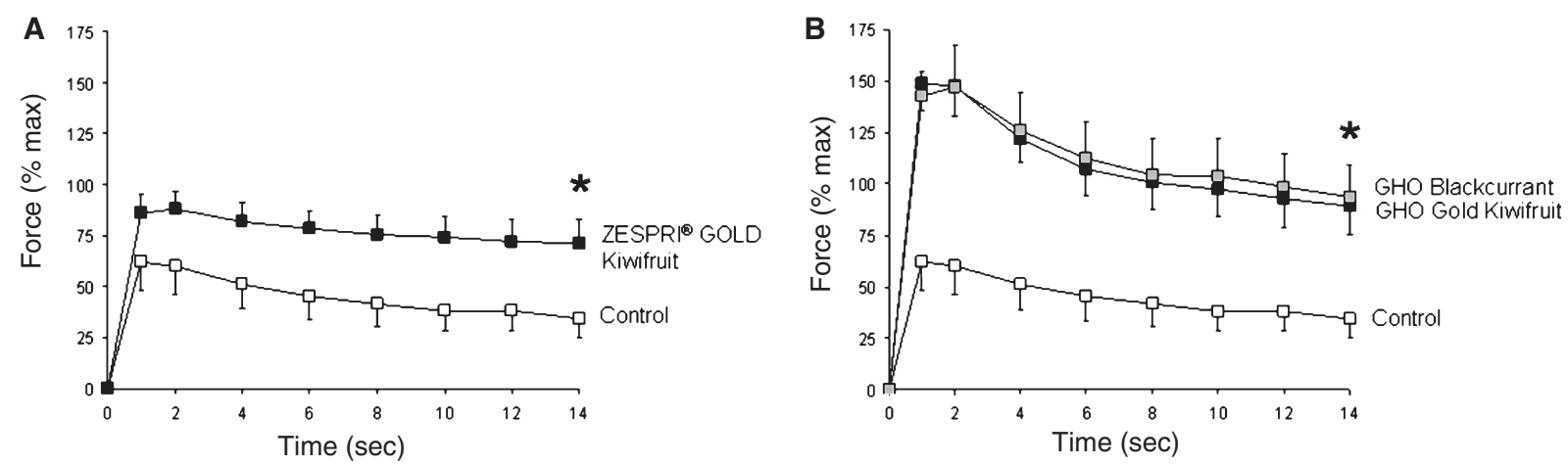

Fig. 3. Effect of fruit extract and juices on fatigue in mouse soleus muscles induced by electrical stimulation. Muscles incubated with (A) ZESPRI ${ }^{\circledR}$ GOLD Kiwifruit (Actinidia chinensis 'Hort16A') phenolic extract ( $5 \mu \mathrm{g} / \mathrm{ml}$, $\square$ control, $\square$ kiwifruit extract), or (B) GHO ZESPRI ${ }^{\circledR}$ GOLD Kiwifruit and blackcurrant juices ( $2 \%, \square$ control, $\square$ kiwifruit, $\square$ blackcurrant) were compared with buffer-only controls. The recorded force was divided by the cross sectional area of the muscle and normalized to the maximum force generated during baseline stimulation. A minimum of five muscles were analysed for each group. Values are means \pm SEM. *Represents $p<0.05$ statistical significance (paired Student $t$-test) between the area under the curve for muscles incubated with buffer-only and fruit extract.

"Natural Quenchers"). Mouse soleus muscles were electrically stimulated ex vivo after 15 min of incubation with the ZESPRI ${ }^{\circledR}$ GOLD Kiwifruit extract $(5 \mu \mathrm{g} / \mathrm{ml})$, or control buffer and tetanic force production was measured over $14 \mathrm{sec}$ (Fig. 3A). Mean peak force in fruit extract exposed muscles was $86.1 \pm 9.7 \%, 23.8 \%$ higher than the peak force in the controls $(62.3 \pm 14.2 \%)$. After the 14 -sec tetanus stimulation the muscles demonstrated fatigue - a decline in muscle performance due to muscle activity. With kiwifruit extract exposure, muscle fatigue was reduced, with the mean force retaining $71.4 \%$ of the peak baseline force, whereas force in the controls dropped to $34.4 \%$. Comparison of the area under the curves highlighted that the ZESPRI ${ }^{\circledR}$ GOLD Kiwifruit mediated protection against fatigue was statistically significant (kiwifruit $1108.5 \pm 147.3$, control $615.2 \pm 151, p<0.05$ ). The commercially prepared ZESPRI ${ }^{\circledR}$ GOLD Kiwifruit and blackcurrant juices mediated a greater enhancement in muscle performance and protection against fatigue (Fig. 3B). Mean peak force in blackcurrant and kiwifruit juice-exposed muscles was $147.1 \pm 20.6 \%$ and $148.9 \pm 13.8 \%$ respectively, $85 \%$ higher than the peak force in controls. After the 14 sec tetanus stimulation, muscle fatigue was reduced by the blackcurrant and kiwifruit juice extracts with the mean force retaining 93.6\% (blackcurrant) and $89.2 \%$ (kiwifruit) of the peak baseline force. Protection against fatigue, analysed as area under the curve, was statistically significant for both blackcurrant $(1546.5 \pm 225.8)$ and kiwifruit juices $(1498.4 \pm 170.1, p<0.05)$.

These data demonstrate that ZESPRI ${ }^{\circledR}$ GOLD Kiwifruit and blackcurrant fruit extracts and juices have an effect on muscle performance, which could be mediated by antioxidant scavenging of exercise-mediated reactive oxygen species (ROS). Active muscles produce excess ROS and it is widely accepted that they play a role in fatigue. During intensive exercise, muscle oxygen consumption increases up to 100-fold, and in parallel, superoxide anion production in mitochondria increases to a level where endogenous ROS scavengers are overwhelmed and hydrogen peroxide diffuses into the muscle cell cytoplasm. Some exogenous antioxidants like $\mathrm{N}$-acetylcysteine (NAC), vitamins $\mathrm{C}$ and $\mathrm{E}$, have been reported to reduce fatigue in isolated muscles, but only NAC has so far been shown to improve fatigue performance in vivo [23].

The fruit compound responsible for the enhanced force production and reduced fatigue in our isolated mouse soleus muscles remains to be elucidated, but it is possible that vitamin $\mathrm{C}$ and/or a variety of polyphenolics present in the fruits and extracts play a role. Indeed, Dorchies and colleagues showed that five weeks of feeding green tea extract and (-)-epigallocatechin gallate (EGCG) to muscular dystrophic mice protected the animals from fatigue and improved muscle force generation [11]. However, while many antioxidants have been shown to provide protection against oxidative stress and fatigue ex vivo, this has not always translated into the in vivo situation. With this in mind we have recently initiated the establishment of animal models of exercise at PFR. Figure 4 shows biochemical data in blood samples taken at various times from mice undertaking an exhaustive uphill running exercise. While not statistically significant, there was a trend towards plasma CK activity (an indicator of muscle tissue damage), 

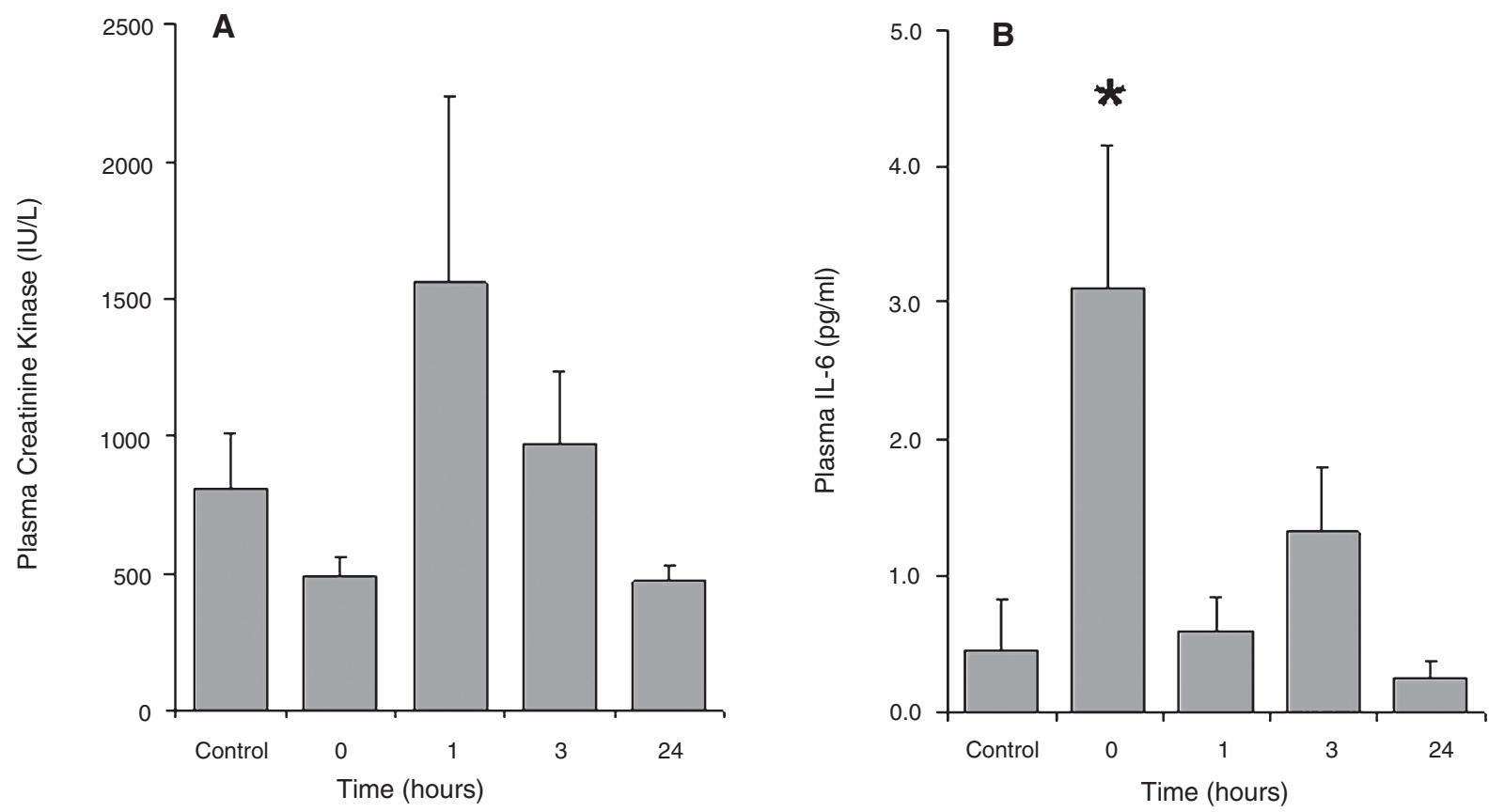

Fig. 4. Markers of stress and inflammation in plasma of exercising mice. Mice performed uphill running on a treadmill until exhaustion and were sacrificed at $0,1,3$ or $24 \mathrm{~h}$ after the exercise. Resting mice functioned as a control group. Blood samples were taken at the time of sacrifice and plasma was isolated. (A) CK activity was expressed as IU/L and (B) IL-6 concentration was expressed as pg/mL. Each group consisted of 12 mice. Values are means \pm SEM. *Represents $p<0.05$ statistical significance (paired Student $t$-test) between control and exercise group.

slightly higher one hour after the exercise, compared with the activity in the control (non-exercised mice Fig. 4A). The release of IL-6 from muscle post exercise is known to be dependent upon both exercise intensity and duration $[6,12]$. Figure 4B shows the plasma levels of IL-6 in exercising mice following our exercise regime. Immediately after the exercise $(0 \mathrm{~h})$, the mean plasma IL-6 concentration significantly increased (7-fold) from $0.45 \pm 0.37 \mathrm{pg} / \mathrm{mL}$ in resting control mice to $3.11 \pm 1.04 \mathrm{pg} / \mathrm{mL}$ in exercised mice $(p<0.05)$. After only 1 hour of recovery, plasma IL-6 concentrations returned to pre-exercise levels. While the exact role of the release of muscle tissue IL-6 is not clear, it is probably released in response to metabolic changes and to oxidative stress and/or tissue damage. It is suspected that IL-6 may be involved in activating aspects of the immune system to assist with tissue repair and recovery. These data also suggests that our uphill running protocol was inducing IL-6 response in exercising mice, without being strenuous or damaging enough to significantly increase CK levels. This is consistent with the findings of others in which no CK increase was observed with an uphill run, while downhill running, which mediates more tissue damage because of its eccentric nature, consistently increased CK in plasma $[4,18]$. Others have utilised exercise routines in animals to evaluate the effects of nutrient supplementation on exercise-induced stress and performance. For example, curcumin [9], green tea extract [21], and Ginseng extract [3] have all been shown to decrease markers of damage, oxidative stress, and inflammation in exercising animals. Our preliminary data are therefore encouraging and in the near future we hope to be able to evaluate the benefits of fruits on stress and inflammation robustly using animal models of exercise.

In humans, exercise can have benefits on the immune system including an ability to positively modulate the acute inflammatory response to a simulated bacterial infection [14]. Furthermore, appropriate fruit extract consumption may assist these health benefits. In a recent study we reported the effect of supplementation with a blackcurrant fruit extract on exercise-induced health benefits [16]. Consumption of the anthocyanin-rich blackcurrant extract prior to a moderate rowing exercise mediated an amelioration of plasma markers of oxidative stress and micro muscle damage, but also complemented the ability of exercise to stimulate an acute inflammatory response to a simulated bacterial infection in ex vivo experiments. These findings could be significant in the augmented activation (by fruit polyphenolics) of 
appropriate adaptive immune responses and of associated positive health benefits derived from moderate and regular exercise. Other studies have evaluated the potential of fruits and/or polyphenolic compound supplementation as antioxidants and reported potential protective effects and benefits to muscle and exercise performance [2, 7, 25]. In contrast however, some studies show that antioxidant supplementation may in fact neutralize the health benefits of regular exercise $[5,24]$ and that in some individuals undergoing long-term strenuous exercise regimes immune suppression can be observed [8].

Further studies are being undertaken at PFR in this area to elucidate the health potential of fruit polyphenolics as appropriate modulators of oxidative stress and inflammation.

\section{Summary}

Our emerging evidence suggests that some fruits (and derived phenolic compounds) offer significant health and wellness potential. Our aim is to utilise unique New Zealand germplasm to unleash opportunities for the creation of premium functional foods (backed by science), to prevent muscle damage/injury, aid recovery, and/or enhance muscular and immune function.

\section{Acknowledgements}

We are grateful for the preparation of the blueberry fruit phenolic extract and the blackcurrant juice by Drs Tony McGhie and Catherine Snelling of PFR. We are grateful for funding support from the New Zealand Foundation for Research, Science and Technology (contract C06X0807, cell and human work) and internal funding from PFR (animal studies).

\section{References}

[1] M. Battino, et al., Bioactive compounds in berries relevant to human health, Nutrition Reviews 67 (2009), S145-S150.

[2] F.P. Bonina, et al., Oxidative stress in handball players: effect of supplementation with a red orange extract, Nutrition Research 25 (2005), 917-924.

[3] A.C. Cabral de Oliveira, A.C. Perez, G. Merino, J.G. Prieto and A.I. Alvarez, Protective effects of Panax ginseng on muscle injury and inflammation after eccentric exercise, Comparative Biochemistry and Physiology. Part C. Toxicology and Pharmacology 130C (2001), 369-377.

[4] M.D. Carmichael, et al., Recovery of running performance following muscle-damaging exercise: Relationship to brain IL-1 beta, Brain Behavior and Immunity 19 (2005), 445-452.

[5] A. Childs, C. Jacobs, T. Kaminski, B. Halliwell and C. Leeuwenburgh, Supplementation with vitamin C and N-acetyl-cysteine increases oxidative stress in humans after an acute muscle injury induced by eccentric exercise, Free Radical Biology and Medicine 31 (2001), $745-753$.

[6] L.H. Colbert, J.M. Davis, D.A. Essig, A. Ghaffar and E.P. Mayer, Tissue expression and plasma concentrations of TNF alpha, IL-1 beta, and IL-6 following treadmill exercise in mice, International Journal of Sports Medicine 22 (2001), 261-267.

[7] D.A.J. Connolly, M.P. McHugh and O.I. Padilla-Zakour, Efficacy of a tart cherry juice blend in preventing the symptoms of muscle damage, British Journal of Sports Medicine 40 (2006), 679-683.

[8] D.M. Cooper, S. Radom-Aizik, C. Schwindt and F. Zaldivar, Dangerous exercise: lessons learned from dysregulated inflammatory responses to physical activity, Journal of Applied Physiology 103 (2007), 700-709.

[9] J.M. Davis, et al., Curcumin effects on inflammation and performance recovery following eccentric exercise-induced muscle damage, American Journal of Physiology-Regulatory Integrative and Comparative Physiology 292 (2007), R2168-R2173.

[10] V.B. Djordjevic, Free radicals in cell biology, in: International Review of Cytology - a Survey of Cell Biology, Vol. 237, edn, Elsevier Academic Press Inc, San Diego, 2004, pp. 57-89.

[11] O.M. Dorchies, S. Wagner, T.M. Buetler and U.T. Ruegg, Protection of dystrophic muscle cells with polyphenols from green tea correlates with improved glutathione balance and increased expression of 67LR, a receptor for (-)-epigallocatechin gallate, Biofactors 35 (2009), 279-294.

[12] C.P. Fischer, Interleukin-6 in acute exercise and training: what is the biological relevance? Exercise Immunology Review 12 (2006), 6-33. 
[13] R.D. Hurst, R.W. Wells, S.M. Hurst, T.K. McGhie, J.M. Cooney and D.J. Jensen, Blueberry fruit polyphenolics suppress oxidative stressinduced skeletal muscle cell damage in vitro, Molecular Nutrition \& Food Research 53 (2009), 1-11.

[14] S.M. Hurst, K.A. Lyall, R.D. Hurst and L.M. Stevenson, Exercise-induced elevation in plasma oxidative generating capability augments the temporal inflammatory response stimulated by lipopolysaccharide, European Journal of Applied Physiology 107 (2009), 61-72.

[15] W.G. Li and A.N. Kong, Molecular mechanisms of Nrf2-mediated antioxidant response, Molecular Carcinogenesis 48 (2009), 91-104.

[16] K.A. Lyall, et al., Short-term blackcurrant extract consumption modulates exercise-induced oxidative stress and lipopolysaccharidestimulated inflammatory responses, American Journal of Physiology-Regulatory Integrative and Comparative Physiology 297 (2009), R70-R81.

[17] A.A. Maglara, A. Vasilaki, M.J. Jackson and A. McArdle, Damage to developing mouse skeletal muscle myotubes in culture: protective effect of heat shock proteins, Journal of Physiology-London 548 (2003), 837-846.

[18] C. Malm, et al., Leukocytes, cytokines, growth factors and hormones in human skeletal muscle and blood after uphill or downhill running, Journal of Physiology-London 556 (2004), 983-1000.

[19] J. Mendez and A. Keys, Density and composition of mammalian muscle, Metabolism-Clinical and Experimental 9 (1960), 184-188.

[20] R.A. Moyer, K.E. Hummer, C.E. Finn, B. Frei and R.E. Wrolstad, Anthocyanins, phenolics, and antioxidant capacity in diverse small fruits: Vaccinium, Rubus, and Ribes, Journal of Agricultural and Food Chemistry 50 (2002), 519-525.

[21] T. Murase, S. Haramizu, A. Shimotoyodome, A. Nagasawa and I. Tokimitsu, Green tea extract improves endurance capacity and increases muscle lipid oxidation in mice, American Journal of Physiology-Regulatory Integrative and Comparative Physiology 288 (2005), R708R715.

[22] T. Nguyen, P. Nioi and C.B. Pickett, The Nrf2-antioxidant response element signaling pathway and its activation by oxidative stress, Journal of Biological Chemistry 284 (2009), 13291-13295.

[23] M.B. Reid, D.S. Stokic, S.M. Koch, F.A. Khawli and A.A. Leis, N-Acetylcysteine inhibits muscle fatigue in humans, Journal of Clinical Investigation 94 (1994), 2468-2474.

[24] M. Ristow, et al., Antioxidants prevent health-promoting effects of physical exercise in humans, Proceedings of the National Academy of Sciences of the United States of America 106 (2009), 8665-8670.

[25] A.L. Rossi, A. Blostein-Fujii and R.A. DiSilvestro, Soy beverage consumption by young men: increased plasma total antioxidant status and decreased acute, exercise-induced muscle damage, Journal of Nutraceuticals, Functional \& Medical Foods 3 (2000), 33-44.

[26] M.A. Skinner, et al., Health benefits of Zespritm gold kiwifruit: effects on muscle performance, muscle fatigue and immune responses, Proceedings of the Nutrition Society of New Zealand 32 (2007), 49-59.

[27] A. Szajdek and E.J. Borowska, Bioactive compounds and health-promoting properties of berry fruits: A review, Plant Foods for Human Nutrition 63 (2008), 147-156.

[28] J.C. Tee, A.N. Bosch and M.I. Lambert, Metabolic consequences of exercise-induced muscle damage, Sports Medicine 37 (2007), $827-836$.

[29] C. Zhan and J. Yang, Protective effects of isoliquiritigenin in transient middle cerebral artery occlusion-induced focal cerebral ischemia in rats, Pharmacological Research 53 (2006), 303-309. 